\title{
Hysteresis Behavior in Shear Rate Dependence of First Normal Stress Difference of Diblock Copolymers in Ordered State near Order-Disorder Transition
}

\author{
Yoshiaki TAKAHASHI ${ }^{1, \dagger}$, Kenta IMAICHI $^{1}$, Masahiro NODA $^{2}$, \\ Atsushi TAKANO ${ }^{2}$, and Yushu MATSUSHITA ${ }^{2}$ \\ ${ }^{1}$ Institute for Materials Chemistry and Engineering, and Department of Molecular \& Materials Sciences, \\ Kyushu University, 6-1 Kasugakoen, Kasuga 816-8580, Japan \\ ${ }^{2}$ Department of Applied Chemistry, Nagoya University, Nagoya 464-8603, Japan
}

(Received November 13, 2006; Accepted March 1, 2007; Published April 17, 2007)

\author{
KEY WORDS Poly(styrene-block-isoprene)s / Poly(styrene-block-2-vinylpyridine)s / Flow- \\ Induced Alignment / Lamellae / First Normal Stress Difference / \\ [doi:10.1295/polymj.PJ2006158]
}

In previous papers, ${ }^{1-5}$ we reported viscoelastic properties and flow-induced structure of symmetric poly(styrene-block-2-vinylpyridine)s (SP) and poly(styrene- $\mathrm{d}_{8}$-block-2-vinylpyridine)s (DP) in a common good solvent, $\alpha$-chloronaphtharene $(\alpha-\mathrm{CN})$ and poly(styrene-block-isoprene)s (SI) and poly(styrene$\mathrm{d}_{8}$-block-isoprene)s (DI) in dioctyl phthalate (DOP) near the order-disorder transition (ODT). Note that viscoelastic properties of components of DP and SP are almost the same. ${ }^{6}$

Characteristic behaviors of viscoelastic properties in the quiescent ordered states are observed in shear rate dependence of first normal stress difference $N_{1}$. That is, $N_{1}$ is proportional to shear rate $\dot{\gamma}$ at low $\dot{\gamma}$ but $N_{1}$ become proportional to $\dot{\gamma}^{2}$ at high $\dot{\gamma}^{1,4}$ An exceptional behavior was observed for DP and SP solutions just above ODT concentration, $C_{\mathrm{ODT}}$. There was no apparent change in the $\dot{\gamma}$ dependence of $N_{1} ; \dot{\gamma}$ dependence of $N_{1}$ was slightly lower than $\dot{\gamma}^{2}$ in the whole range of tested shear rate. ${ }^{4}$

Flow-induced structure of $\mathrm{DP}^{2-4}$ and $\mathrm{DI}^{5}$ solutions are studied by small angle neutron scattering under steady shear flow (flow-SANS). The data for both samples are compared in connection with the difference of viscoelastic properties of components. ${ }^{5}$ It is observed for DI solutions ${ }^{5}$ that parallel and perpendicular alignments of lamellae, in which lamellar normals are parallel to shear gradient and vorticity directions, respectively, coexist at low $\dot{\gamma}$ and the latter become dominant at high $\dot{\gamma}$ at a certain concentration $C$. The perpendicular alignments become dominant with decrease of $C$. For DP solutions, coexistence of parallel and perpendicular alignments are only observed at low $\dot{\gamma}$ for relatively high $C,{ }^{2}$ while only per- pendicular alignment was observed at lower $C$ near the ODT. ${ }^{2-4}$

From above results, it was concluded that the parallel alignment becomes minor near the ODT and/or high $\dot{\gamma}$ and such tendency is pronounced for the samples whose viscosity ratio of components is close to 1. ${ }^{5}$ At the same time, it was reported that the degree of perpendicular alignment is primarily determined by effective interaction parameters, $(\chi N)_{\text {eff }}$ and reduced shear rates, $\dot{\gamma} / \dot{\gamma}^{*}$, irrespective of the components' viscosity ratio, where $\dot{\gamma}^{*}$ is the critical $\dot{\gamma}$ for non-Newtonian behavior. ${ }^{5}$

Time slice measurements of SANS after the cessation of flow revealed that anisotropic scattering peak reflecting the perpendicular alignment still remains more than the half of initial value after $3 \mathrm{~h}$ rest for a DP solution $2 \mathrm{wt} \%$ higher than $C_{\mathrm{ODT}}{ }^{7}$ and a DI solution $0.3 \mathrm{wt} \%$ higher than $C_{\mathrm{ODT}},{ }^{8}$ while that for a DP solution $0.5 \mathrm{wt} \%$ above $C_{\mathrm{ODT}}$ disappear instantaneously after the cessation of flow. ${ }^{3}$ These differences in stability of aligned structure for DP and DI solutions may not be simply explained by the difference in fluctuation and/or relaxation times. Consideration of other factors such as dynamics of grains may be needed. The difference in the viscosity ratio in the components might have a certain role. Though the reasons are not clarified, it is apparent that the aligned lamellae of DP solutions are much unstable near the ODT. Note that above studies are carried out with successive increase of $\dot{\gamma}$ and the relaxation measurements are started from the highest $\dot{\gamma}$. Under successive decrease of $\dot{\gamma}$, another difference in the behavior of $N_{1}$ between two different diblock samples is expected, as mentioned below.

${ }^{\dagger}$ To whom correspondence should be addressed (Tel/Fax: +81-92-583-8822, E-mail: ytak@mm.kyushu-u.ac.jp). 
Table I. Molecular characteristics of samples

\begin{tabular}{ccccc}
\hline $\begin{array}{c}\text { Sample } \\
\text { Code }\end{array}$ & $10^{-4} M_{\mathrm{w}}$ & $\begin{array}{c}M_{\mathrm{w}} / M_{\mathrm{n}} \\
\text { (by GPC) }\end{array}$ & $\phi_{\mathrm{PS}}$ & $\begin{array}{c}C_{\mathrm{ODT}} \\
(\text { wt } \%)\end{array}$ \\
\hline SP-12 & 42.2 & 1.03 & 0.52 & 11.1 (at $30^{\circ} \mathrm{C}$ ) \\
$\mathrm{DP}-20$ & 36.0 & 1.08 & 0.53 & 12.0 (at $30^{\circ} \mathrm{C}$ ) \\
DI-10 & 11.5 & 1.12 & 0.5 & 27.5 (at $27^{\circ} \mathrm{C}$ ) \\
\hline
\end{tabular}

$M_{\mathrm{w}}$ : Weight averaged molecular weight. $M_{\mathrm{n}}$ : Number averaged molecular weight. $\phi_{\mathrm{PS}}$ : Volume fraction of polystyrene.

When $\dot{\gamma}$ is lowered from the $\dot{\gamma}$ region where lamellae are well aligned, $N_{1}$ may become proportional to $\dot{\gamma}^{2}$ even at low $\dot{\gamma}$ (hysteresis behavior) for the samples with stable aligned structure, whereas $N_{1}$ may not be proportional to $\dot{\gamma}^{2}$ at low $\dot{\gamma}$ for the solutions with unstable aligned structure (SP and DP close to ODT). In this paper, we examine the above speculations by measurements of $N_{1}$ and flow-SANS with successive increase and decrease of shear rate.

\section{EXPERIMENTAL}

Block copolymer samples used are the same ones (SP-12, DP-20 and DI-10) as used in the previous studies. ${ }^{2-5,7-9}$ Their molecular characteristics are tabulated in Table I. The solvents and measuring temperatures $\left(\alpha-\mathrm{CN} ; 30.0 \pm 0.5^{\circ} \mathrm{C}\right.$ for SP-12 and DP-20, DOP; $27.0 \pm 0.5^{\circ} \mathrm{C}$ for DI-10) are also the same as those in previous studies.

Viscoelastic properties of SP-12 and DP-20 solutions are measured with a Mechanical Spectrometer RMS800 of Rheometric Scientific while those of DI10 solution are measured with a MCR300 rheometer of Anton Parr. Cone-plate geometry of $5 \mathrm{~cm}$ diameter and $0.04 \mathrm{rad}$ cone-angle is used in these measurements. The measurements are conducted with successive increase of $\dot{\gamma}$, followed by decrease of the $\dot{\gamma}$. Hereafter, we simply call the former and the latter type of measurements as increase and decrease measurements, respectively. Only $\dot{\gamma}$ dependence of $N_{1}$ is discussed since the effects of structural change (alignment of lamellae) is clearly observed in the behavior of $N_{1}$ but not for shear stress.

Flow-induced structure of microdomains in decrease measurements are examined by flow-SANS measurements using a couette type flow cell, ${ }^{10}$ attached to the SANS-U spectrometer at the Neutron Scattering Laboratory of the ISSP, The University of Tokyo in Tokai, Japan. The wavelength $\lambda$, the diameter of incident neutron beam and the sample to detector distance are $0.7 \mathrm{~nm}, 5 \mathrm{~mm} \phi$, and 4 and $8 \mathrm{~m}$, respectively. Only the data obtained by so-called "through view" measurement, in which the incident neutron beam is in the direction normal to the surface of the couette cell, are analyzed in this work, after the cor-
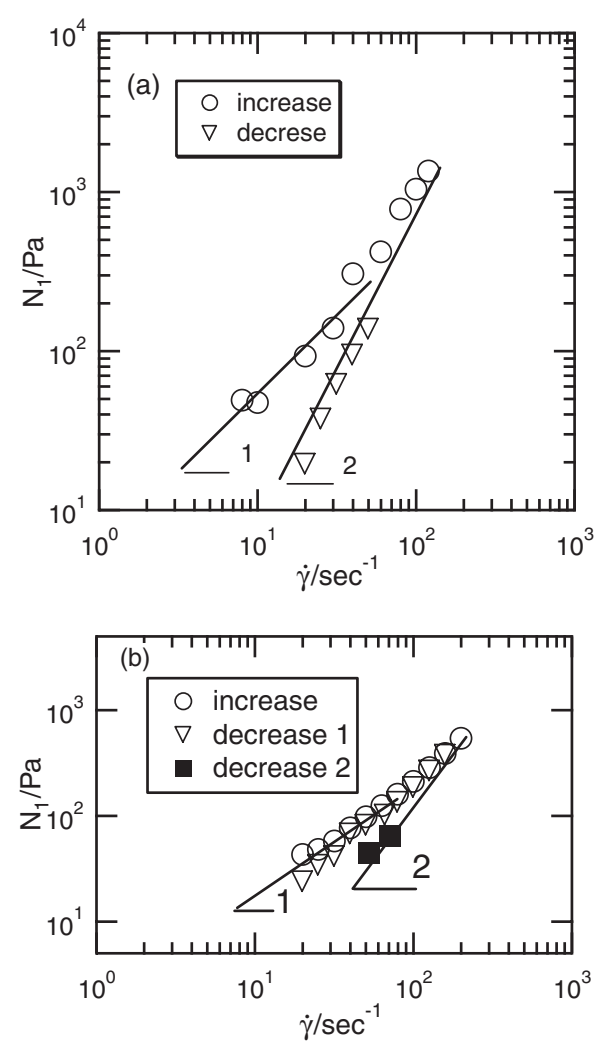

Figure 1. Double logarithmic plots of $N_{1}$ against $\dot{\gamma}$ for SP-12 (a): $18.8 \mathrm{wt} \%$ and (b): $15.0 \mathrm{wt} \%$ solutions. Symbols are denoted in the figure.

rection for the incoherent scattering of the solvent and empty cell. Sector averaged intensities within sectors of $\pm 5^{\circ}$ arc in the vertical and horizontal directions on the detector, $I_{\mathrm{Y}}$ and $I_{\mathrm{X}}$ are obtained as a function of wave vector $q=4 \pi \sin (\theta / 2) / \lambda$, where $\theta$ is scattering angle.

\section{RESULTS AND DISCUSSIONS}

Figure 1 shows double-logarithmic plots of $N_{1} v s . \dot{\gamma}$ obtained by increase and decrease measurements for SP-12, 15.0 and $12.6 \mathrm{wt} \%$ solutions. It is apparent that $N_{1}$ is proportional to $\dot{\gamma}$ at low $\dot{\gamma}$ range, while it becomes proportional to $\dot{\gamma}^{2}$ at higher $\dot{\gamma}$ range in the increase measurement, in consistent with previous studies. ${ }^{1,4}$ In the decrease measurement, $\dot{\gamma}^{2}$ dependence of $N_{1}$ is maintained at lower $\dot{\gamma}$ range for $15.0 \mathrm{wt} \%$ solution, while $\dot{\gamma}$ dependences of $N_{1}$ in the successive increase and decrease (decrease 1) measurements are almost the same for SP-12, $12.6 \mathrm{wt} \%$ solution as expected. For the latter solution, the data obtained by the decrease of $\dot{\gamma}$ from the highest one to the respective $\dot{\gamma}$ (decrease 2) are also shown. In this case, $\dot{\gamma}^{2}$ dependence of $N_{1}$ is maintained in contrast to the data obtained in the successive decrease of $\dot{\gamma}$. These results imply that the alignment of lamellae and corresponding value of $N_{1}$ is largely affected by the prior flow. 

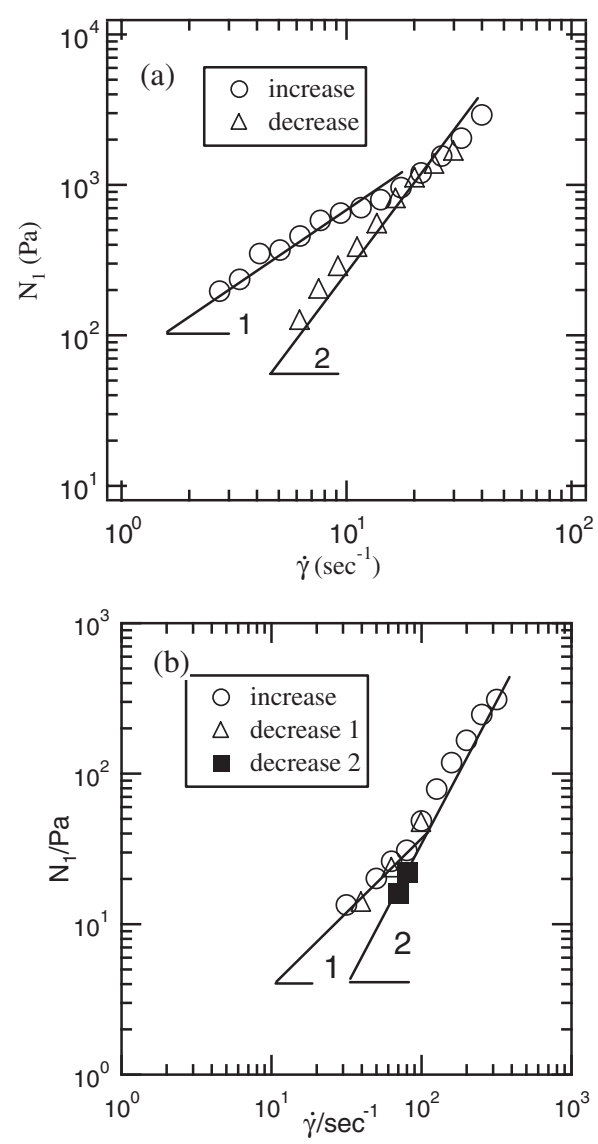

Figure 2. Double logarithmic plots of $N_{1}$ against $\dot{\gamma}$ for (a): DI10, $27.8 \mathrm{wt} \%$ solution and (b): DP-20, $14 \mathrm{wt} \%$ solution. Symbols are denoted in the figure.

Figure 2 shows double-logarithmic plots of $N_{1} v s$. $\dot{\gamma}$ for DI-10, $27.8 \mathrm{wt} \%$ and DP-20, $14.0 \mathrm{wt} \%$ solutions. A clear hysteresis behavior is observed for the DI-10 solution, which is only $0.3 \mathrm{wt} \%$ higher than $C_{\text {ODT }}$. Note that flow-induced microphase separation and alignment of lamellae, same level as this solution, takes place for DI solutions in the disordered states ${ }^{5}$ so that the data in the decrease measurement without hysteresis cannot be observed for DI solutions. The feature of Figure 2a (DP-20) is practically the same as that of SP-12, $12.6 \mathrm{wt} \%$ solution, though the data are scarce at low $\dot{\gamma}$ range since the magnitude of $N_{1}$ become close to the lower measuring limit.

To clarify the relation between hysteresis behavior and degree of alignment, changes of peak intensities $I_{\mathrm{Y}}$ and $I_{\mathrm{X}}$ in the decrease measurements for DP-20, $14.0 \mathrm{wt} \%$ solution are obtained and plotted against $q$ in Figure 3. As reported previously, ${ }^{5} I_{\mathrm{X}}$ was about one order lower than the values at rest and insensitive of $\dot{\gamma}$. It is clear that $I_{\mathrm{Y}}$ in the decrease measurement $\left(\dot{\gamma}: 200 \rightarrow 50 \mathrm{~s}^{-1}\right.$, corresponding to decrease 2 in Figure $2 b$ ) are higher than those observed in the increase measurement. On the other hand, the data corresponding to successive decrease (decrease 1 in

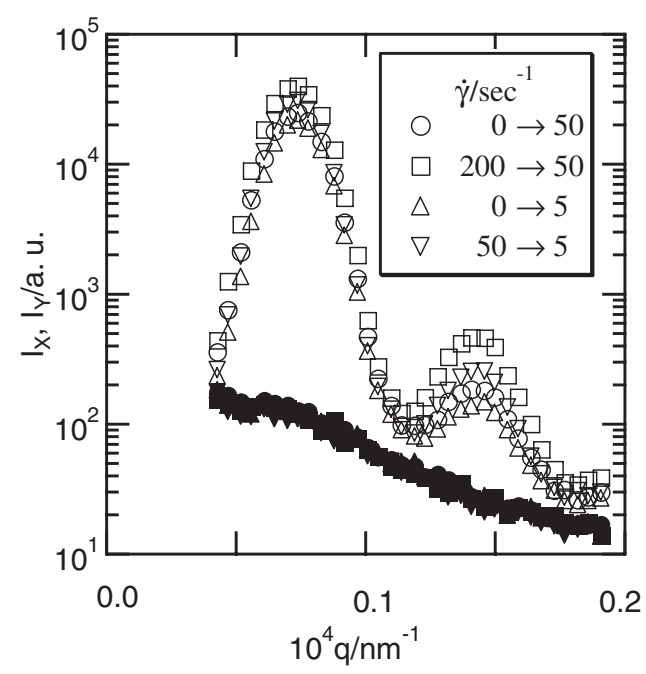

Figure 3. Plots of $I_{Y}$ (open symbols) and $I_{\mathrm{X}}$ (Filled symbols) in increase and decrease measurements for (a) DP-20, $14.0 \mathrm{wt} \%$ solution. Symbols are denoted in the figure.

Figure $2 \mathrm{~b}$ ) are almost the same as those observed in the increase measurement. Because of the experimental difficulties, flow-SANS measurements for DI-10, $27.8 \mathrm{wt} \%$ solution are limited at $\dot{\gamma}<1.0 \mathrm{~s}^{-1}$, lower than those in the rheological measurements (Figure 2a). In this case too, $I_{Y}$ in the increase and decrease measurements are almost the same. Though the tested $\dot{\gamma}$ are not exactly the same as those in the rheological measurements, we can conclude that the hysteresis behavior of $N_{1}$ is due to the well aligned structure maintained even after the decrease of $\dot{\gamma}$.

Finally, we comment on the origin of $\dot{\gamma}^{2}$ dependence of $N_{1}$. The $\dot{\gamma}$ range employed in the rheological measurements for DI is much higher than that in flow-SANS measurements, whereas those for DP are comparable. The perpendicular alignment of lamellae is dominant at the tested $\dot{\gamma}$ range for DP-20. The parallel alignment of lamellae coexisted for DI-10 at the tested $\dot{\gamma}$ range of flow-SANS. ${ }^{5}$ The parallel alignment of lamellae decreases with increase of $\dot{\gamma}$. Therefore, we speculate that the perpendicular alignment of lamellae is also dominant at the $\dot{\gamma}$ range where $\dot{\gamma}^{2}$ dependence of $N_{1}$ is observed for DI-10 so that the $\dot{\gamma}^{2}$ dependence of $N_{1}$ of lamellar-forming diblock copolymers is strongly correlated to the perpendicular alignment of the lamellae.

\section{REFERENCES}

1. Y. Takahashi, M. Noda, N. Ochiai, and I. Noda, Polymer, 37, 5943 (1996).

2. S. Kitade, N. Ochiai, Y. Takahashi, I. Noda, Y. Matsushita, A. Karim, A. I. Nakatani, H. Kim, and C. C. Han, Macromolecules, 31, 8083 (1998).

3. Y. Takahashi, M. Noda, S. Kitade, and I. Noda, J. Phys. 
Chem. Solids, 60, 1343 (1999).

4. Y. Takahashi, M. Noda, S. Kitade, K. Matsuoka, Y. Matsushita, and I. Noda, Polym. J., 37, 894 (2005).

5. Y. Takahashi, M. Naruse, Y. Akazawa, A. Takano, and Y. Matsushita, Polym. J., 37, 900 (2005).

6. Y. Takahashi, N. Ochiai, Y. Matsushita, and I. Noda, Polym. J., 28, 1065 (1996).
7. M. Noda, Master Course Thesis, Nagoya University, 1998.

8. Y. Naruse, Master Course Thesis, Nagoya University, 2000.

9. Y. Takahashi, S. Kitade, M. Noda, N. Ochiai, I. Noda, M. Imai, and Y. Matsushita, Polym. J., 30, 388 (1998).

10. Y. Takahashi, M. Noda, M. Naruse, T. Kanaya, H. Watanabe, T. Kato, M. Imai, and Y. Matsushita, Nihon Reoroji Gakkaishi, 28, 187 (2000). 